\title{
In Response: Does Coadministration of Transforaminal Epidural Steroid Injection with Sedation Improve Patient Satisfaction?
}

We would like to thank the authors for their interest in our article, "Does coadministration of transforaminal epidural steroid injection with sedation improve patient satisfaction? A prospective randomized clinical study" (1). We believe that this provides us with an opportunity to further discuss the topics of sedation for interventional techniques.

In our study, we compared the satisfaction of patients in the groups undergoing transforaminal epidural steroid injection (TFESI) with or without sedation due to unilateral single nerve root involvement associated with lumbar disc herniation. As pointed out by the authors, the presence of unblinded patients to the intervention might have resulted in bias in our results, which can be regarded as a limitation. However, it would be technically unreasonable and unethical to expose the patients in a blind manner to an intervention under sedation. As the patients received intervention either under sedation or unsedated, no comparison was able to be made. Therefore, a comparison between patients who received sedation and those who did not seems to be controversial when the desire of the patient was questioned whether he or she demanded the same technique or not. However, low patient satisfaction and low treatment adherence owing to discomfort or dislike may affect the results, although the treatment is solely effective (2). Thus, it would be a reasonable question to be answered as it indicates patient satisfaction indirectly, but not directly among those undergoing this intervention with or without sedation. As such, certain patients may not be willing to receive TFESI under the same technique, whether sedated or unsedated, due to postprocedural adverse effects, which can be considered a predictive factor for procedure-related patient dissatisfaction. However, we believe that further studies comparing TFESI with and without sedation would pave the way for new insights into this topic.

It is well known by most anesthesiologist and intensive care medicine specialists that prone position improves airway mechanics $(3,4)$. Patients, when positioned prone, have an increase in functional residual capacity, improved ventilation/perfusion matching, and subsequently improved oxygenation as a consequence of changes in the distribution of both ventilation and perfusion all through the lungs during anesthesia management (3). Also, prone positioning diminishes the low ventilation/perfusion ratio areas, sustains a more uniform distribution of alveolar stress and better right-ventricular hemodynamics, and therefore it is a well-defined treatment for respiratory distress in intensive care units (4). Most of the anesthesia drugs, all intravenous opioids and intravenous benzodiazepines, propofol, inhaler agents, and even ketamine in high doses makes respiratory depression with different mechanisms (5). Lodenius et al (5) reported that even dexmedetomidine sedation diminishes both hypoxic and hypercapnic regulation of breathing. Therefore, as all intravenous sedatives cause dose-dependent depression, it is the art of anesthesiology that we perform every day to titrate the anesthetic drugs we use and utilize their beneficial effects while avoiding their side effects. In this study, we increase efficiency in the art of dose titrating by using the Observer's Assessment of Alertness/Sedation scale, and we can suggest this scale for less experienced sedation personnel to diminish any possible side effects.

\author{
Savas Sencan, MD \\ Department of Physical Medicine and Rehabilitation \\ Division of Pain Medicine \\ Marmara University, Istanbul, Turkey \\ Muhsin Yazıcıoğlu Cd No:10, 34899 \\ Pendik/Istanbul, Turkey \\ Ipek Saadet Edipoglu, MD \\ Department of Physical Medicine and Rehabilitation \\ Division of Pain Medicine \\ Marmara University, Istanbul, Turkey \\ Muhsin Yazıcıoğlu Cd No:10, 34899 \\ Pendik/Istanbul, Turkey \\ Serhad Bilim, MD \\ Department of Physical Medicine and Rehabilitation \\ Division of Pain Medicine \\ Marmara University, Istanbul, Turkey \\ Muhsin Yazıcıoğlu Cd No:10, 34899 \\ Pendik/İstanbul, Turkey
}


Osman Hakan Gunduz, MD

Department of Physical Medicine and Rehabilitation

Division of Pain Medicine

Marmara University, Istanbul, Turkey

Muhsin Yazıcıoğlu Cd No:10, 34899

Pendik/Istanbul, Turkey
Ipek Saadet Edipoglu, MD

Department of Physical Medicine and Rehabilitation

Division of Pain Medicine

Marmara University, Istanbul, Turkey

Muhsin Yazıcıoğlu Cd No:10, 34899

Pendik/İstanbul, Turkey

E-mail: dripeks@yahoo.com

\section{RefEREnCES}

1. Sencan S, Edipoglu IS, Bilim S, Gunduz $\mathrm{OH}$. Does coadministration of transforaminal epidural steroid injection with sedation improve patient satisfaction? A prospective randomized clinical study. Pain Physician 2019; 22:E287-E294.

2. Prabhu V, Alukal JP, Laze J, Makarov DV, Lepor $\mathrm{H}$. Long-term satisfaction and predictors of use of intracorporeal injections for post-prostatectomy erectile dysfunction. J Urol 2013; 189:238-242.

3. Edgcombe H, Carter K, Yarrow S. Anaesthesia in the prone position. $\mathrm{Br}$ J Anaesth 2008; 100:165-183.

4. Ohnson NJ, Luks AM, Glenny RW. Gas exchange in the prone posture. Respir
Care 2017; 62:1097-1110.

Lodenius A, Ebberyd A, Hårdemark Cedborg A, et al. Sedation with dexmedetomidine or propofol impairs hypoxic control of breathing in healthy male volunteers: A nonblinded, randomized crossover study. Anesthesiology 2016; 125:700715 . 